\title{
Analyse und Auswertung von Besonderheiten und Problemen bei der Konstruktion einer Serienvorrichtung im Rahmen einer praxisnahen Aufgabenstellung für die Lehre
}

\author{
Norbert Miersch, Gero Busse, Jörg Fricke, Alexander Stengl
}

\section{Einleitung}

Der Kontakt und die Zusammenarbeit mit Klein- und Mittelständischen Unternehmen in der Umgebung der Technischen Fachhochschule Wildau ist eine wichtige Wissensquelle zur Bereicherung von Lehre und Forschung. Dabei werden praxisnahe Problemstellungen im Hauptstudium in den belegbegleitenden Fächern der Spezialisierungsrichtung Maschinenbau von Studenten zunehmend angestrebt.

Im Zuge der sich verschärfenden Arbeitsmarktsituation kann der Nachweis solcher Aktivitäten bei der Bewerbung durchaus förderlich sein. Basierend auf bestehenden Kontakten zur Firma Tema Fischer GmbH in Berlin Rudow war es möglich, für zwei Studenten eine praxisnahe Belegaufgabe fuir das Lehrgebiet Vorrichtungskonstruktion zu formulieren. Dabei konnten der Aufgabenumfang und die zeitliche Terminstellung mit der Firma entsprechend dem Lehrplan angepasst werden.

\section{Zielstellung}

Vorgesehen war, zwei Serienvorrichtungen für die mechanische Bearbeitung eines Getriebegehäusehinterteiles des „Porsche Cayenne“ mit Hilfe eines 3D-CADSystems zu konstruieren. Vereinbart wurde dabei die Erstellung der Zeichnungssätze, der Dokumentationen sowie einer abschließenden Präsentation.

Das Werkstuick „Getriebegehäusehinterteil“ sollte durch den Einsatz der beiden Vorrichtungen in zwei Aufspannungen auf einem Bearbeitungszentrum „MORI SEIKI SH-633“ bearbeitet werden. Der zeitliche Rahmen und das Arbeitsvolumen mussten in zehn Semesterwochen mit jeweils vier Semesterwochenstunden zuzüglich Selbststudienzeit durch den Einsatz der zwei Studenten bewältigt werden. Außerdem war es aufgrund des vorliegenden Datenformates der 3D-CAD-Daten des Getriebegehäusehinterteiles notwendig, Schnittstellenuntersuchungen durchzufuihren. Diese eigenständige Aufgabenstellung sollte im parallel laufenden Lehrgebiet „Rechnerintegrierter Betrieb“ von den zwei Studenten bearbeitet werden.

Eine wichtige Aufgabe bestand darin, das optimale Datenformat für die Vorrichtungskonstruktion und damit im Zusammenhang das zweckmäßigste verfuigbare 3DCAD-System zu ermitteln.

\section{Aussagen zur Herstellung des Werkstückes}

Das Werkstuick „Getriebegehäusehinterteil“, das in seiner Serienvariante mit 20000 Stück pro Jahr in der Firma Tema Fischer GmbH hergestellt werden soll, ist ein Druckgussteil aus dem Werkstoff GD-AlSi9Cu3. Die mechanische Teilebearbeitung ordnet sich dabei in die Herstellung der Fertigteile wie folgt ein (Abbildung 1):

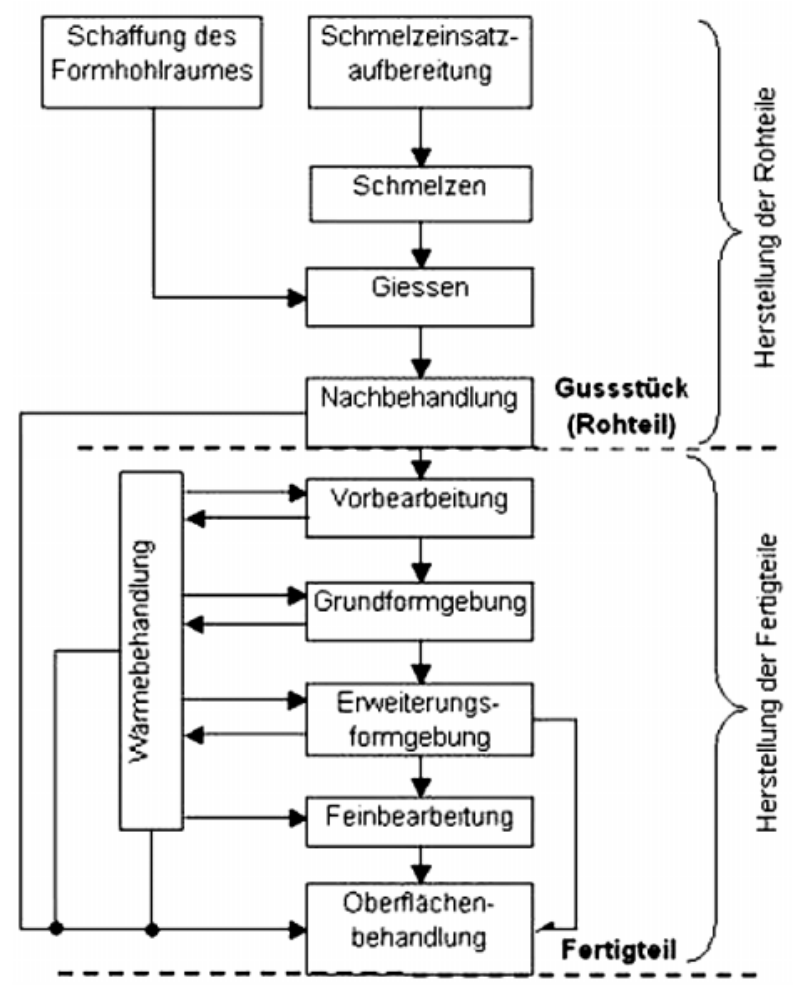

Abb. 1: Abgrenzung von Roh- und Fertigteil im Teilefertigungsprozess von Gussstücken [1]

In einer modernen mechanischen Werkstatt wie in der Firma Tema Fischer GmbH wird eine Komplettbearbeitung der Werkstuicke angestrebt. Das bedeutet, dass alle notwendigen spanenden Arbeitsschritte auf einer einzigen Werkzeugmaschine durchgefuihrt werden.

Eine solche Verfahrensweise ist auch beim Getriebegehäusehinterteil möglich. Durch das Vorgießen von Formelementen, wie beispielsweise der Grund- oder Durchgangsbohrungen, der Verrippungen, der Nuten und der Schlitze, konnte bereits am Gussteil selbst eine hohe Annäherung der Rohteilgeometrie an die Fertigteilgeometrie erfolgen (Abbildung 2). 


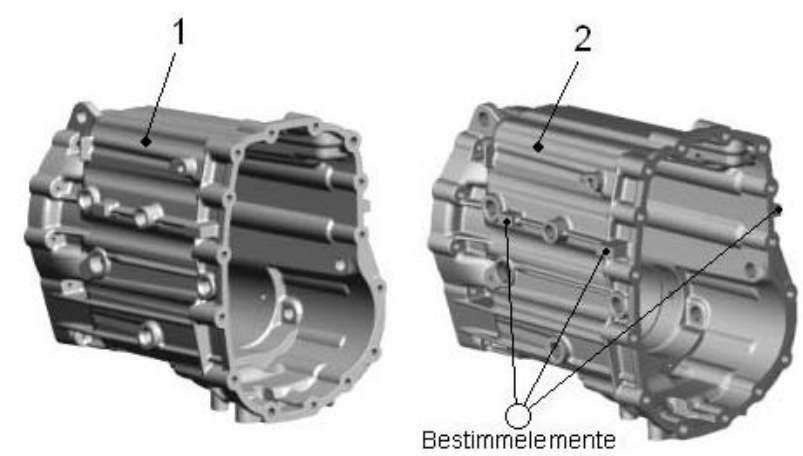

Abb. 2: Getriebegehäusehinterteil für den Porsche Cayenne (1 - Druckgussrohteil in perspektivischer Darstellung; 2 - Fertigteil in perspektivischer Darstellung)

Der Ablauf in Abbildung 1 kann demzufolge durch Zusammenlegung der Schritte Vorbearbeitung, Grundformgebung, Erweiterungsformgebung und Feinbearbeitung fuir das Getriebegehäusehinterteil präzisiert werden. Für Druckgussteile aus Aluminiumguss wird allgemein nach [2] von folgenden technisch- technologischen Richtwerten ausgegangen (siehe Tabelle 1):

\begin{tabular}{|r|l|}
\hline Bearbeitungszugaben $:$ & $0,3 \mathrm{~mm}$ bis $0,5 \mathrm{~mm}$ \\
\hline Aushebeschrägen $:$ & $1^{\circ}$ bis $5^{\circ}$ \\
\hline Mindestwanddicken $:$ & $1 \mathrm{~mm}$ bis $3 \mathrm{~mm}$ \\
\hline Maßabweichungen $:$ & ca. $\pm 0,2 \mathrm{~mm}$ \\
\hline Mindestradien : & bis $1 \mathrm{~mm}$ möglich \\
\hline
\end{tabular}

Tab. 1: Richtwerte für Druckgussteile aus Aluminiumlegierungen nach [2]

Die mechanische Bearbeitung des Getriebegehäusehinterteiles macht es notwendig, Grund-, Durchgangsund Gewindebohrungen sowie Flächen in einer nach Fertigteilzeichnung vorgeschriebenen Qualität herzustellen. Dafür werden die spanenden Bearbeitungsverfahren Bohren, Senken, Reiben, Zirkularfräsen, Gewindeformen, Planfräsen und Ausdrehen angewandt. Durch den Einsatz eines Bearbeitungszentrums ist eine Komplettbearbeitung des Getriebegehäusehinterteiles in zwei Aufspannungen mit einem entsprechenden Werkzeugsatz möglich.

Die Entwicklung eines serientauglichen Gussrohteiles fuir das Getriebegehäusehinterteil dauert nach Untersuchungen [3] ca. zwei Jahre. Dem Serienteil läuft bereits nach einem halben Jahr in einer begrenzten Stückzahl ein Prototyp voraus. Das Gussrohteil fuir die Serienfertigung wird dabei auf Grundlage der Fertigteilzeichnung in der Gießerei konstruiert [3]. Es erfolgen umfangreiche Absprachen zwischen dem Getriebekonstrukteur und dem Rohteilkonstrukteur in der Gießerei.

Das Rohteil ist durch eine Vielzahl von Parametern gekennzeichnet. Um die Wichtigsten zu nennen, sind dies die Formteilung, Kerne, Bearbeitungszugaben, Aushebeschrägen, Gussradien, vorgießbare Formelemente, Markierungen der Anguss-, Speisungs- und Entliuftungsstellen und die Definition von Spannpunkten sowie Aufund Anlageflächen zur nachfolgenden mechanischen Bearbeitung [1].
Gerade die Definition der Auf- und Anlageflächen stellt für die technologische Basierung der Bearbeitungsoperationen wie Abspanen, Beschichten, Montage- und Prüfoperationen eine wichtige Grundlage dar. Spannpunkte dienen zu unmittelbaren Kraftübertragung durch Spannmittel am Gussteil [1]. Durch das Festlegen zweckmäßiger Auf- und Anlageflächen bzw. Spannpunkte kann der Fertigungsaufwand reduziert werden.

Dieser Sachverhalt wurde auch beim Getriebegehäusehinterteil berüicksichtigt. Um ein Verspannen und ein zeitaufwändiges Vermitteln einzusparen, wurden Aufund Anlageflächen berücksichtigt (Abbildung 3).
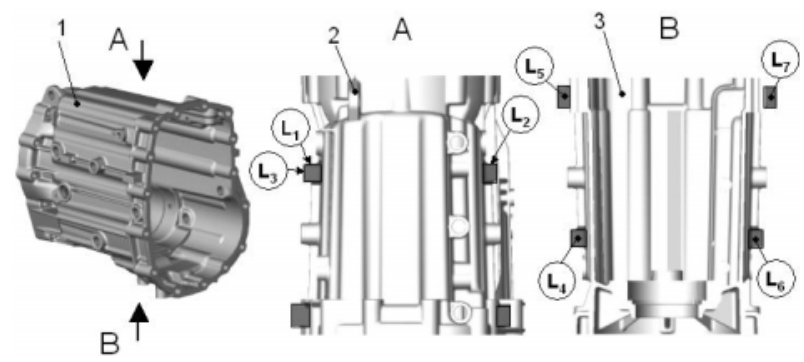

Abb. 3: Definition der An- und Auflageflächen durch den Getriebekonstrukteur (1 - Fertigteil in perspektivischer Darstellung; 2 - Ansicht der An- und Auflageflächen von oben (Ansicht A); 3 - Ansicht der An- und Auflageflächen von unten im Schnitt (Ansicht B); L1, L2, L3 - Fest- und Anlageflächen für Rohkontrolle und Erstbearbeitung; L4, L5, L6 - Fest- und Auflageflächen für Rohkontrolle und Erstbearbeitung; L7 - bewegliche Auflagefläche für die Erstbearbeitung (Stütze))

Um jedoch zweckentsprechende Auf- und Anlageflächen zum Bestimmen ${ }^{1}$ in einer Vorrichtung zu erhalten, sind Absprachen des Getriebekonstrukteurs mit dem Vorrichtungskonstrukteur erforderlich. In den wenigsten Fällen wird dieser Sachverhalt berïcksichtigt, so dass in der mechanischen Bearbeitung ein hoher Kostenaufwand entsteht.

\section{Besonderheiten zur Vorrichtungskonstruktion}

Im Folgenden wird auf die Besonderheiten bei der Vorrichtungskonstruktion für die erste Aufspannung der Komplettbearbeitung des Getriebegehäusehinterteiles auf dem Bearbeitungszentrum „MORI SEIKI SH-633“ eingegangen.

\subsection{Anforderungen an die Vorrichtung der ersten Aufspannung}

In Abstimmung mit der Firma Tema Fischer GmbH konnte zur Konstruktion der Vorrichtung eine Anforderungsliste erstellt werden (Tabelle 2).

Zur Herstellung der ersten Getriebegehäusehinterteile wurde bereits ein Vorrichtungsprototyp genutzt. Nach einer Analyse der Fertigung mit diesem Prototyp und dem Erfahrungswissen von Spezialisten der Firma Tema Fischer GmbH konnte diese Anforderungsliste nach Tabelle 2 fuir eine Serienvorrichtung erstellt werden. 


\begin{tabular}{|c|c|}
\hline Anforderungen & Begründung \\
\hline Einfachspannung & $\begin{array}{l}\text { Da sich die Vorrichtung im Bearbeitungszentrum auf einem Drehtisch befindet, } \\
\text { müssen alle vier Seiten zugänglich sein. Eine Vorrichtung die das gewährleistet, } \\
\text { ist bei dieser Teilegröße nicht zu realisieren. }\end{array}$ \\
\hline Eine Bedienstelle & $\begin{array}{l}\text { Wichtig bei größeren Stückaahlen ist die Reduzierung der Haupt- und } \\
\text { Nebenzeiten. Durch eine Bedienstelle werden die Nebenzeiten gering gehalten. }\end{array}$ \\
\hline Hydraulisches Spannsystern & $\begin{array}{l}\text { Zum Spannen von Gussteilen wurden bereits Erfahrungen mit hydraulischen } \\
\text { Spannelementen gesammelt. Durch die Funktion dieser Spannelemente können } \\
\text { die Nebenzeiten ebenfalls gering gehalten werden. Hier wurde vorzugsweise auf } \\
\text { den Einsatz von Schwenkspannern verwiesen. }\end{array}$ \\
\hline $\begin{array}{r}\text { Vermeidung von } \\
\text { Verspannungen (Offset) }\end{array}$ & $\begin{array}{l}\text { Um Verspannungen zu vermeiden, ist es zwingend notwendig, die festgelegten } \\
\text { Auf- und Anlageflächen, sowie Spannflächen des Konstrukteurs zu nutzen. } \\
\text { Außerdem können hierdurch komplizierte Toleranzuntersuchungen vermieden } \\
\text { werden. }\end{array}$ \\
\hline Schwingungsdämpfung & $\begin{array}{l}\text { Durch den Einsatz des Vorrichtungsprototypen waren bei der Bearbeitung } \\
\text { erhebliche Schwingungen und Geräusche zu verzeichnen. Die neuen } \\
\text { Vorrichtungen sollen das Schwingungsproblem durch einen entsprechenden } \\
\text { Vorrichtungsgnundkörper aus EN-GJL und eine kompaktere bzw. steifere } \\
\text { Auslegung eingrenzen. }\end{array}$ \\
\hline $\begin{array}{l}\text { Betrachtung der } \\
\text { Punktauflage }\end{array}$ & $\begin{array}{l}\text { Das Bestimmen des Gussteiles auf den Vorrichtungsflächen soll durch eine } \\
\text { Punktauflage erfolgen. Die herbei entstehenden Spannmarken drücken sich in } \\
\text { die vom Konstrukteur vorgesehenen Auflageflächen ein. Sie sind zulässig, da sie } \\
\text { auf die Funktion des Getriebes keinen Einfluss haben. Jedoch kann das } \\
\text { Absenken des Gussteiles Einfluss auf die Toleranzen haben. Dieser Einfluss } \\
\text { (Hert'zsche Pressung) ist wenn möglich zu überprüfen. }\end{array}$ \\
\hline Überwachung. Sicherheit & $\begin{array}{l}\text { Überwachung der Spannkräfte durch Kopplung eines Kraftsensors mit der } \\
\text { Steuerung der Maschine. Bei größeren Spannkraftschwankungen ist die } \\
\text { Werkzeugmaschine (BAZ) außer Betrieb zu setzten. Die Komplettbearbeitung } \\
\text { des Getriebegehäuses wird durch den Einsatz mehrerer Werkzeuge realisiert. } \\
\text { Diese Sonderwerkeuge stellen einen emeblichen Kostenfaktor dar. } \\
\text { Werkzeugbruch und Maschinenzerstönung soll dadurch verhindert werden. }\end{array}$ \\
\hline
\end{tabular}

Tab. 2: Anforderungsliste zur Vorrichtungskonstruktion

\subsection{Die Bedeutung von 3D-CAD-Werkstïckdaten bei der Vorrichtungskonstruktion}

Für eine 3D-CAD-Vorrichtungskonstruktion ist es zweckmäßig, die 3D-CAD-Werkstuickdaten des Getriebegehäusehinterteiles zu nutzen. Das ist beispielsweise zur Überprïfung von Kollisionen vorteilhaft, die beim Einlegen oder Herausnehmen des Werkstuickes aus der Vorrichtung auftreten können.

Das Bestimmen und die eventuell erforderlichen Toleranzuntersuchungen werden in der gängigen Praxis anhand vorhandener 2D-CAD-Fertigteilzeichnungen im Papierformat durchgefuihrt. Gegenuiber einer 3D-CADDarstellung enthalten diese Zeichnungen zusätzliche geometrische und technologische Informationen. Es können beispielsweise neben dem Werkstoff wichtige Maß-, Form-, Lage- und Oberflächentoleranzen abgelesen werden.

Zum Getriebegehäusehinterteil lagen 2D-Zeichnungen fuir das Rohteil und das Fertigteil im Papierformat vor. Die Daten des Getriebegehäusehinterteiles wurden zusätzlich der Firma Tema Fischer GmbH vom Getriebekonstrukteur im Pro/ENGINEER-Format übermittelt. Ein entsprechendes 3D-CAD-System war an der TFH Wildau und der Firma Tema Fischer GmbH nicht verfuigbar. Auf Anfrage beim Getriebekonstrukteur war es möglich, mehrere neutrale Datenformate zu erhalten. Diese wurden gesondert im Lehrgebiet „Rechnerintegrierter Betrieb“ auf ihre Verwendbarkeit überprüft.
Aufgrund dieser Untersuchungen stellte sich für die Vorrichtungskonstruktion das 3D-CAD-System Solid Works2002 als günstigstes heraus. Einen qualitativen Vergleich der verschiedenen Datenformate zum entsprechenden an der TFH Wildau verfuigbaren 3D-CAD-System zeigt Tabelle 3 .

\begin{tabular}{|c|c|c|c|}
\hline & $\begin{array}{l}\text { IGES } \\
\text { ( } v 5.3 \text { ) }\end{array}$ & $\begin{array}{c}\text { STEP } \\
\text { (AP214) }\end{array}$ & $\begin{array}{c}\text { VDAFS } \\
(\vee 2)\end{array}$ \\
\hline Catia V5 & $\begin{array}{c}K \\
\text { genūgend } \\
\text { Flächenorientierung }\end{array}$ & $\underset{\text { sehr gut }}{\uparrow}$ & $\underset{\text { ungenügend }}{\downarrow}$ \\
\hline SolidWorks2002 & $\begin{array}{c}\mathbf{K} \\
\text { genūgend } \\
\text { Flächenoriertierung }\end{array}$ & $\underset{\text { sehr gut }}{\uparrow}$ & $\begin{array}{c}\downarrow \\
\text { ungenügend }\end{array}$ \\
\hline Mechanical Desktop 6 & $\begin{array}{c}\uparrow \\
\text { sehr gut }\end{array}$ & $\underset{\text { ungenügend }}{\downarrow}$ & $\underset{\text { ungenügend }}{\downarrow}$ \\
\hline Inventor 5 & $\underset{\text { ungenügend }}{\downarrow}$ & $\begin{array}{c}\uparrow \\
\text { sehr gut }\end{array}$ & $\underset{\text { ungenügend }}{\downarrow}$ \\
\hline
\end{tabular}

Tab. 3: Qualitativer Vergleich ausgewählter 3D-CAD-Systeme hinsichtlich ihrer Datenaufnahme und -verarbeitung über ausgewählte neutrale Schnittstellen

\subsection{Lösung wichtiger Probleme bei der Werkstückbestimmung}

Wie bereits im Punkt 3 erwähnt, wurden durch den Getriebekonstrukteur Auf- und Anlageflächen am Werkstück definiert (siehe Abbildung 3). Auf diese Flächen wurde ein Referenznullpunkt festgelegt. Alle für die mechanische Bearbeitung zutreffenden Maße sind auf diesen Referenznullpunkt bezogen und entsprechend toleriert. Werden die vorgesehenen Werkstuickbestimm- 
flächen mit den Vorrichtungsbestimmflächen in Übereinstimmung gebracht, liegt ein Bestimmfehler zweiter Ordnung vor. Bei diesem Bestimmfehler ist es nicht notwendig eine Toleranzuntersuchung durchzufuihren, um die Genauigkeit der zu bearbeitenden Formelemente in Abhängigkeit von den Vorrichtungsbestimmflächen am Werkstück nachzuweisen [4].

Aufgrund einer günstigeren Vorrichtungsvariante war das Bestimmen auf einige dieser Auf- und Anlageflächen nicht möglich. Die vom Getriebekonstrukteur vorgesehenen Bezugsebenen ${ }^{2} \mathrm{~L}_{4}, \mathrm{~L}_{5}$ und $\mathrm{L}_{6}$ in Abbildung 3 konnten mit den Bestimmebenen ${ }^{3}$ nicht in Übereinstimmung gebracht werden (Abbildung 4/1). Durch den nun vorliegenden Fehler erster Ordnung [4] war es notwendig, eine Toleranzuntersuchung durchzufuihren (Abbildung 4/2).

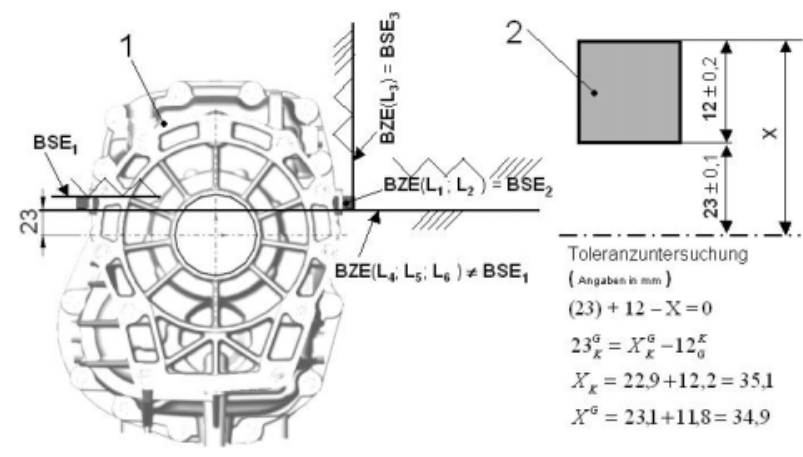

Abb. 4: Werkstückaufbereitung und Toleranzuntersuchung am Getriebegehäusehinterteil (Werkstück) (1 - Werkstückaufbereitung; 2 - Toleranzkette; BSE - Bestimmebene; BZE - Bezugebene)

Das Ergebnis der Toleranzrechnung weist ein größeres Kleinstmaß $\left(X_{K}=35,1 \mathrm{~mm}\right)$ als das Größtmaß $\left(X_{G}=34,9\right.$ $\mathrm{mm})$ aus. Um diesen Bestimmfehler auszugleichen, wurden folgende Möglichkeiten betrachtet und miteinander verglichen:

1. Gewährleistung der Übereinstimmung von Bezugsebene und Bestimmebene durch Änderung der Lage der Bestimmebene. Damit verbunden ist eine für die Serienfertigung unwirtschaftliche Vorrichtungsvariante.

2. Gewährleistung der Übereinstimmung von Bezugsebene und Bestimmebene durch Änderung der Bemaßung durch den Getriebekonstrukteur (Veränderung des Bezuges der Bemaßung zu den Auflageflächen). Damit verbunden ist eine Änderung der Getriebekonstruktion und der Konstruktion des Druckgusswerkzeuges.

3. Veränderung der Maßtoleranzen durch den Getriebekonstrukteur. Das hat eine Veränderung des Gussrohteiles und des Druckgusswerkzeuges zur Folge.

4. Beibehaltung der Bemaßung und der Maßtoleranzen. Zusage einer höheren Genauigkeit in der Rohteilfertigung unter Berïcksichtigung des Werkzeugverschleißes.

In Absprache mit dem Werkzeugkonstrukteur der Druckgussform, dem Gießer und der Firma Tema Fischer GmbH konnte die vierte Möglichkeit gewählt werden. Die vorgesehene Vorrichtungsvariante konnte somit berücksichtigt werden.

\subsection{Einsatz moderner Spannelemente im klassischen Spannkonzept}

Um ein sicheres, verzugsfreies und reproduzierbares Spannen ${ }^{4}$ des Werkstuickes in der Vorrichtung zu gewährleisten, müssen schrittweise folgende Kriterien berücksichtigt werden:

1. Ermittlung der vorhandenen Schnitt- und Vorschubkräfte (Kraftangriffspunkt, Betrag und Wirkungsrichtung). Berücksichtigung der ungünstigsten Bearbeitungsaufgabe für jede Koordinatenrichtung [5]. In diesem Zusammenhang kann gleichzeitig eine Überprüfung der vorhandenen Maschinenleistung erfolgen.

2. Ermittlung des Korrekturfaktors für elastisches bzw. starres Spannen und Korrektur der Schnittkraft- und Vorschubkräfte [6].

3. Festlegung der Spannstellen am Werkstiick.

4. Berechnung der notwendigen Spannkräfte. Dazu ist das Aufstellen eines Kräfteplanes zweckmäßig.

5. Auswahl bzw. Berechnung der Spannelemente. Nutzung von entsprechenden Herstellerkatalogen. ${ }^{5}$

6. Nachrechnung gefährdeter Bauteilquerschnitte.

7. Überprüfung auf Spannmarken am Werkstïck.

Zur Ermittlung der vorhandenen Schnitt- und Vorschubkräfte wurde das NC-Programm des Bearbeitungszentrums „MORI SEIKI SH-633“ ausgewertet. Unter Berücksichtigung der ungünstigsten Bearbeitungssituation (Bearbeitung der größten Bohrung) wurde daraus die Mindestspannkraft für jede Spannstelle errechnet. Im Ergebnis waren geringe Spannkräfte erforderlich. Begründet werden kann dies durch die geringen Bearbeitungszugaben am Rohteil (siehe Tabelle 1) und geringen Vorschuibe bei der Bearbeitung.

Als Spannelemente wurden doppelt wirkende hydraulische Schwenkspanner eingesetzt (Firma Römheld). Die Schwenkspanner werden beim Spannen und Entspannen hydraulisch betätigt und verfügen über einen Spann- und einen Schwenkhub. Durch das Ausschwenken der Spanner beim Entspannen wird ein leichtes Be- und Entladen der Vorrichtung möglich. Das Bedienen aller Schwenkspanner durch die Hydraulikanlage ist an einer Bedienstelle möglich.

Um das Überbestimmen des Werkstückes zu vermeiden, aber gleichzeitig eine stabile und schwingungsarme Werkstücklage zu gewährleisten, wurde ein hydraulisches Abstiitzelement eingesetzt (Firma Römheld). Die Steuerung dieses Stiitzelementes ist zeitversetzt im Zusammenhang mit einem hydraulischen Schwenkspanner durch den Einsatz eines Stromregelventils im Hydraulikkreislauf möglich (Abbildung 5).

\subsection{Berücksichtigung weiterer Einflussgrößen}

Aufgrund der vorliegenden Erfahrungen beim Einsatz des Vorrichtungsprototypen (siehe auch Punkt 4.1) traten bei der Bearbeitung des Gehäusehinterteiles selbsterregte Schwingungen auf. Dieser Sachverhalt kam 


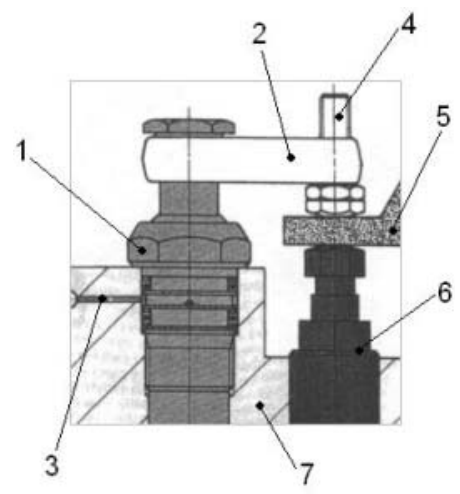

Abb. 5: Kombinierter Einsatz hydraulischer Stütz- und Spannelemente (1 doppelt wirkender Schwenkspanner; 2 - Schwenkeinheit des Schwenkspanners; 3 -Hydraulikleitung; 4 -verstellbares Druckelement; 5 - Werkstück; 6 - hydraulisches Abstützelement)

besonders durch die entstehenden Geräusche bei der Fertigung zum Tragen. Der Einfluss wurde bei der Konstruktion des Vorrichtungsgrundkörpers berïcksichtigt. Dieser wurde besonders massiv und schwer mit $160 \mathrm{~kg}$ zu 9,3 kg Werkstiickmasse sowie dem Werkstoff EN-GJL ausgefuihrt.

In Abstimmung mit der Firma Tema Fischer GmbH wurden zum Bestimmen Punktauflagen verwendet. Eine entsprechende Spannmarkenbetrachtung ist hierbei nicht notwendig, da es in jedem Fall zu Spannmarken am Werkstuick kommt und sie die Funktion des Getriebes nicht beeinflussen. Sie können nach Absprache mit dem Getriebekonstrukteur am Werkstuick verbleiben.

Der nach Anforderungsliste zu untersuchende Einfluss der Eindrücktiefe auf die Fertigungstoleranzen wird von der Firma Tema Fischer GmbH empirisch untersucht. Eine analytische Betrachtung wurde aufgrund der Kompliziertheit des Problems (plastische und elastische Analyse) und des damit verbundenen hohen Zeitaufwandes nicht durchgefuihrt. Diese Entscheidung wurde mit der Firma Tema Fischer GmbH abgestimmt. Das Sicherheitskonzept nach Anforderungsliste (Tabelle 2) wird von der Firma Tema Fischer GmbH selbst ausgefuihrt.

\subsection{Die Ausführung der Vorrichtung für die erste Aufspannung}

Der Aufbau der Vorrichtung wird in Abbildung 6 dargestellt. Hierbei ist zu beachten, dass für den Schwenkspanner noch keine aktualisierten 3D-CAD-Daten durch den „Anbieter“ zur Verfuigung standen, obwohl der komplette Schwenkspanner bereits im Handel erhältlich ist. Bezuiglich der Schwenkarme müssten die Zeichnungen der Vorrichtung dann noch ergänzt werden.

Im Weiteren wird die Funktion der Vorrichtung erläutert. Im Ausgangszustand sind die Schwenkspanner (Positionen 6.1 bis 6.4 in Abb. 6) vor dem Einlegen der Werkstiicke zur Seite geschwenkt. Die Klemmung der hydraulische Stuitze (Positionen 2 in Abb. 6) ist entriegelt.

Bei der Bestuickung der Vorrichtung wird zunächst das Werkstuick auf die Bestimmelemente der Vorrichtung gelegt. Dabei werden die Auf- und Anlageflächen (siehe

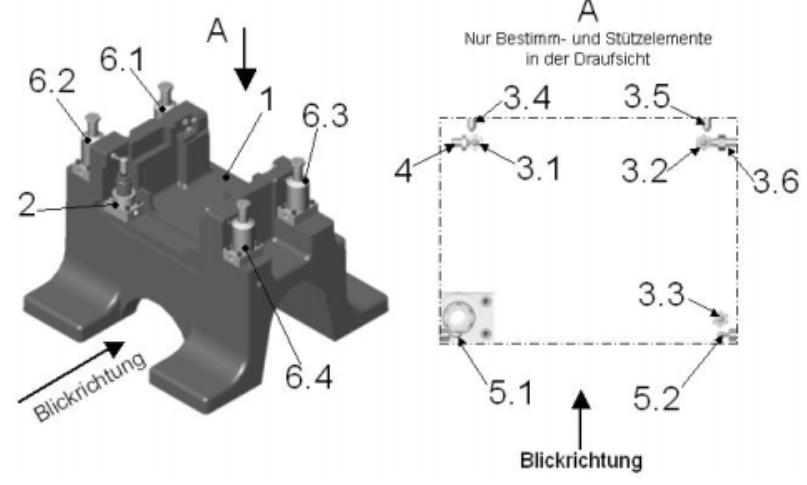

Abb. 6: Vorrichtung für die mechanische Bearbeitung von Getriebegehäusehinterteilen des Porsche Cayenne (1 - Vorrichtungsgrundkörper; 2 - hydraulisch betätigte Stütze; 3.1 ; 3.2; 3.3; 3.4; 3.5; 3.6 - Kugelauflage M8; 4 Druckstift M8; 5.1; 5.2 - Seitendruckstück M12; 6.1; 6.2; 6.3; 6.4 Schwenkspanner)

Abb. 4 und Abb. 6) des Werkstuickes mit den Bestimmebenen $\mathrm{BSE}_{1}, \mathrm{BSE}_{2}$ und $\mathrm{BSE}_{3}$ über Kugelauflagen (Positionen 3.1 bis 3.6 in Abb. 6) an der Vorrichtung in Berührung gebracht. Durch das Eigengewicht des Werkstuickes wird dies selbstständig an die Kugelauflagen (Positionen 3.1 bis 3.3 in Abb. 6, Bestimmebene BSE $_{1}$ im Abb. 4) gedrüickt. Hierbei werden dem Werkstiick drei Freiheitsgrade entzogen. Durch die Seitendruckstiicke (Positionen 5.1 bis 5.2 in Abb. 6) wird das Werkstiick gleichzeitig mit $150 \mathrm{~N}$ an die Kugelauflagen (Positionen 3.4 und 3.5 in Abb. 6, Bestimmebene $\mathrm{BSE}_{2}$ in Abb. 4) gedrückt. Es werden dem Werkstiick weitere zwei Freiheitsgrade entzogen. Der federnde Druckstift (Position 4 in Abb. 6) sorgt für die Lagesicherung des Werkstückes an einer weiteren Kugelauflage (Position 3.6 in Abb. 6, Bestimmebene $\mathrm{BSE}_{3}$ in Abb. 4). Hierbei wird dem Werkstuick ein Freiheitsgrad entzogen. Danach erfolgt der Einsatz der ersten drei Schwenkspanner (Positionen 6.1 bis 6.3 in Abb. 6). Sie Spannen das Werkstuick gegen die Kugelauflagen (Positionen 3.1 bis 3.3 in Abb. 6, Bestimmebene $\mathrm{BSE}_{1}$ in Abb. 4).

Anschließend wird die hydraulische Stuitze (Position 2 in Abb. 6) soweit an die Auflagefläche des Werkstückes (Bestimmebene $\mathrm{BSE}_{1}$ in Abb. 4) herangefahren, bis eine Federkraft von ca. $15 \mathrm{~N}$ auf die Auflagefläche des Werkstuicks wirkt. Die Stuitze wird danach hydraulisch geklemmt. Es erfolgt das Einschwenken des vierten Spanners (Position 6.4 in Abb. 6) und das Fixieren der letzten Spannstelle.

Bei der Entnahme der Werkstuicke aus der Vorrichtung werden durch die hydraulische Steuerung die Schwenkspanner (Positionen 6.1 bis 6.4 in Abb. 6) gleichzeitig nach oben gefahren und schwenken kollisionsfrei um $90^{\circ}$ nach außen. Die hydraulisch betätigte Stuitze (Position 2 in Abb. 6) wird gleichzeitig entsperrt. Die Werkstuicke können aus der Vorrichtung entnommen werden.

Die Vorrichtung wird an einem Schalter der Hydraulikanlage betätigt. Der Ablauf des Spannens erfolgt automatisch durch die Nutzung von Wege- und Stromregelventilen im Hydraulikkreislauf. In Abbildung 7 wird die Vorrichtung mit dem Werkstuick „Getriebegehäusehinterteil“" dargestellt. 


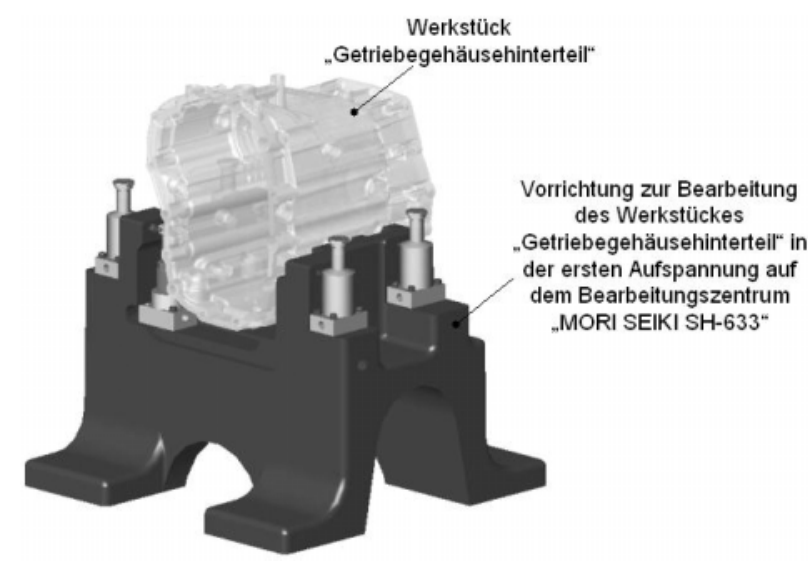

Abb. 7: Vorrichtung zur mechanischen Bearbeitung mit Werkstück „Getriebegehäusehinterteil“" des Porsche Cayenne

\section{Analyse der Ergebnisse}

Die vorgegebene Zielstellung unter Punkt 2 wurde erfuillt. Es wurden die Vorrichtungen für beide Aufspannungen konstruiert. Die Firma Tema Fischer GmbH konnte die vorgelegten Ergebnisse nutzen, um die Fertigung der Vorrichtungen einzuleiten. Es erfolgte eine erfolgreiche Verteidigung der Lösung durch beide Studenten im Unternehmen.

Beide Vorrichtungen wurden mit Hilfe des 3D-CAD-Systems SolidWorks konstruiert. Dabei konnten die Werkstuickdaten des Getriebekonstrukteurs konvertiert und genutzt werden.

\section{Zusammenfassung und Ausblick}

Der vorliegende Artikel beschreibt ausgehend von den Betrachtungen zum Werkstuick „Getriebegehäusehinterteil“ Besonderheiten und Probleme bei der Konstruktion einer Serienvorrichtung für die erste Aufspannung der Komplettbearbeitung auf einem Bearbeitungszentrum. Durch die Zusammenarbeit mit erfahrenen Spezialisten aus der Fertigung war es möglich, einige Besonderheiten und praxisorientierte Probleme unter Berüicksichtigung des bekannten Konstruktionsablaufes (Bestimmen und Spannen) zu beschreiben.

Der Artikel macht deutlich, dass trotz intensiver Absprachen zwischen Fertigteilkonstruktion und Rohteilfertigung während der Entwicklungsphase eines Serienteiles die Besonderheiten der mechanischen Bearbeitung nicht berüicksichtigt wurden. Dieser Sachverhalt wurde bei der Betrachtung der An- und Auflageflächen am Getriebegehäusehinterteile des Porsche Cayenne erkannt.

Weiterfuihrend ist geplant, das erarbeitete Wissen im Rahmen des Lehrgebietes „Vorrichtungskonstruktion“ an die Studenten zu vermitteln und durch neue Aufgabenstellungen mit dem Kooperationspartner zu erweitern. Aufgrund einer sehr gut funktionierenden Zusammenarbeit mit der Firma Tema Fischer GmbH ist die Bearbeitung weiterer Problemstellungen möglich. Der Erfolg wird jedoch in erster Linie von der Motivation der Stu- denten beeinflusst, denn die Bearbeitung einer solchen Aufgabe erfordert ein hohes Maß an persönlichen Engagement verbunden mit einem überdurchschnittlichen Zeitaufwand.

\section{Danksagung}

Die Autoren danken an dieser Stelle Herrn Professor Kuchling und Frau Dipl.-Ing. (FH) Gabriele Wille für die sehr gute Zusammenarbeit, ohne die die Schnittstellenuntersuchungen zu den 3D-CAD-Daten nicht möglich gewesen wären.

\section{Anmerkungen}

1 Das Bestimmen ist das Einordnen des Werkstücks in eine eindeutige, fuir die Durchfuihrung der Arbeitsverrichtung erforderliche Lage [4]. Die Vorgehensweise beim Bestimmen wird als Methode des Entzuges der Freiheitsgrade bezeichnet [5].

2 Bezugsebenen sind die Ebenen, auf die der Konstrukteur des Werkstïcks die maßlichen Festlegungen bezieht [4].

3 Bestimmebenen sind die Ebenen, die durch den Kontakt zwischen Werkstiick und Bestimmelementen der Vorrichtung entstehen [4].

4 Spannen ist das sichere Festhalten der bereits bestimmten Werkstücke oder Werkzeuge während der Fertigung [4].

5 Zur Lieferung von Bestimm- und Spannelementen sowie Normteilen für den Vorrichtungsbau gibt es „Anbieter“. Diese präsentieren sich über Kataloge im Papierformat und über das Internet. Bei der Nutzung des Internets besteht dabei die Möglichkeit, Daten der Vorrichtungselemente in einem maschinenlesbaren Datenformat herunterzuladen. Es werden hier die gängigen Datenformate wie IGES, DXF, STEP, DWG, SAT angeboten. Einige ausgewählte Anbieter sind RÖMHELD, NORELEM und HASCO.

\section{Literaturverzeichnis}

[1] Miersch, N.: Rechnerunterstiitzte Generierung von Gussrohteilen aus Eisengusswerkstoffen, Dissertationsschrift, Otto-von-Guericke-Universität Magdeburg 2002.

[2] Ambos, E.; Soethe, M.; Hofmann, I.: CITIM - Datenbank Gießereitechnologie. Ein Hilfsmittel zur Gestaltung von Bauteilen aus metallischen Gusswerkstoffen, Gießereipraxis (1994) 15/16, S. 405-411.

[3] Swat, H.: Analyse des technologischen Gussteiledurchlaufs vom Eingang der Anfrage bis zur Serienfertigung in der Kloth-Senking Metallgießerei Hildesheim, Praktikumsbericht 2002, Technische Fachhochschule Wildau

[4] Fronober, M.; Henning, W.; Thiel, H.; Wiebach, H.: Vorrichtungen. Gestalten, Bemessen, Bewerten, VEB Verlag Technik, Berlin 1982.

[5] Trummler, A.; Wiebach, H.: Vorrichtungen der Produktionstechnik, Verlag Friedrich Vieweg \& Sohn, Braunschweig/Wiesbaden 1994.

[6] Perovic, B.: Werkzeugmaschinen und Vorrichtungen. Berechnung und Konstruktion, Carl Hanser Verlag, München/Wien 1999. 


\section{Autoren}

\section{Dr.-Ing. Norbert Miersch}

Technische Fachhochschule Wildau

Fachbereich Ingenieurwesen/Wirtschaftsingenieurwesen

Telefon +493375 508-161

E-Mail: miersch@hrz.tfh-wildau.de

\section{Dipl.-Ing. (FH) Gero Busse}

TEMA-Fischer $\mathrm{GmbH}$

Kanalstraße 7 A, 12357 Berlin

Telefon +49306606066

E-Mail: g.busse@tema-fischer.de

\section{Jörg Fricke}

Finowstraße 6, 10247 Berlin

Telefon +493029494661

E-Mail: jfricke@igw.tfh-wildau.de

\section{Alexander Stengl}

Blumberger Damm 3, 16306 Casekow OT Wartin

Telefon +493333165105

E-Mail: a.stengl@gmx.net 